PAPER

\title{
Epidemiology of benign paroxysmal positional vertigo: a population based study
}

\author{
M von Brevern, A Radłke, F Lezius, M Feldmann, T Ziese, T Lempert, H Neuhauser
}

See Editorial Commentary, p 663

J Neurol Neurosurg Psychiatry 2007;78:710-715. doi: 10.1136/jnnp.2006.100420

See end of article for authors' affiliations

......................

Correspondence to: Dr Michael von Brevern Neurologische Klinik, Charité, Campus VirchowKlinikum, Augustenburger Platz 1, 13353 Berlin, Germany; michael.von_brevern@ charite.de

Received 20 June 2006 Revised 19 October 2006 Accepted 19 October 2006 Published Online First 29 November 2006
Objectives: To examine the prevalence and incidence, clinical presentation, societal impact and comorbid conditions of benign paroxysmal positional vertigo (BPPV) in the general population.

Methods: Cross-sectional, nationally representative neurotological survey of the general adult population in Germany with a two stage sampling design: screening of 4869 participants from the German National Telephone Health Interview Survey 2003 (response rate 52\%) for moderate or severe dizziness or vertigo, followed by validated neurotological interviews $(n=1003$; response rate $87 \%$ ). Diagnostic criteria for BPPV were at least five attacks of vestibular vertigo lasting $<1 \mathrm{~min}$ without concomitant neurological symptoms and invariably provoked by typical changes in head position. In a concurrent validation study $(n=61)$ conducted in two specialised dizziness clinics, BPPV was detected by our telephone interview with a specificity of $92 \%$ and a sensitivity of $88 \%$ (positive predictive value $88 \%$, negative predictive value $92 \%$ ).

Results: BPPV accounted for $8 \%$ of individuals with moderate or severe dizziness/vertigo. The lifetime prevalence of BPPV was $2.4 \%$, the 1 year prevalence was $1.6 \%$ and the 1 year incidence was $0.6 \%$. The median duration of an episode was 2 weeks. In $86 \%$ of affected individuals, BPPV led to medical consultation, interruption of daily activities or sick leave. In total, only $8 \%$ of affected participants received effective treatment. On multivariate analysis, age, migraine, hypertension, hyperlipidaemia and stroke were independently associated with BPPV.

Conclusion: BPPV is a common vestibular disorder leading to significant morbidity, psychosocial impact and medical costs.
B enign paroxysmal positional vertigo (BPPV) is probably the most common cause of vestibular vertigo accounting for approximately $20-30 \%$ of diagnoses in specialised dizziness clinics. ${ }^{12}$ The evolution of highly effective positioning manoeuvres ${ }^{3}$ has made BPPV the most successfully treatable cause of vertigo.

Thus far, however, the epidemiology of BPPV in the general population is not known. The aim of this study was to estimate the prevalence and incidence of BPPV in the general adult population in Germany and to characterise the clinical features, impact and risk factors of BPPV in a nationally representative sample.

\section{METHODS}

\section{Study population}

A cross-sectional neurotological survey was conducted emanating from the German National Telephone Health Interview Survey 2003 (GNT-HIS). Details on the survey have been published recently. ${ }^{5}$ In brief, the GNT-HIS 2003 is a nationally representative health survey of the adult population in Germany with computer assisted telephone interviews $(\mathrm{n}=8318)$ covering various aspects of diseases, including risk factors, quality of life, health care utilisation and socioeconomic status. The response rate of the GNT-HIS 2003 was 52.3\%. Participants for the neurotological survey were screened by the following question: Did you ever experience moderate or severe dizziness or vertigo? Out of a simple random sample of 4869 participants of the GNT-HIS, 4077 gave consent for further interview and 1157 fulfilled the inclusion criteria for the neurotological survey (history of moderate or severe dizziness/ vertigo, and still valid telephone number) and 1003 interviews were completed (response rate $87 \%$ ).

\section{Neurotological survey}

The neurotological survey comprised a validated telephone interview designed to differentiate between vestibular vertigo and non-vestibular dizziness and to identify specific syndromes (BPPV, Menière's disease, acute unilateral vestibular loss, migrainous vertigo and orthostatic dizziness). The interviews were performed by two medical student interviewers (FL and MF) who were extensively trained in a neurological dizziness clinic during a period of 1 year. All interviews were discussed with an experienced neurotologist ( $\mathrm{MvB}, \mathrm{AR}, \mathrm{TL}, \mathrm{HN})$ who also supervised $10 \%$ of the interviews in person.

\section{Diagnostic criteria}

Vestibular vertigo and BPPV were diagnosed according to criteria developed for this survey through piloting and validation in a specialised dizziness clinic (table 1). Our definition of the term "vestibular vertigo" required a history of vertigo with an illusion of rotation or provocation of vertigo by positional changes of the head or dizziness with accompanying symptoms including oscillopsia, nausea and imbalance. Secondary BPPV was diagnosed when participants reported a history of acute unilateral vestibular loss or head trauma within 6 months of the onset of BPPV or intubation within 3 days beforehand. Criteria for acute unilateral vestibular loss were a history of an isolated episode of prolonged spontaneous rotational vertigo lasting several days without concurrent neurological symptoms. Furthermore, a history of migraine was diagnosed according to the criteria of the International Headache Society (IHS). ${ }^{6}$

Abbreviations: BPPV, benign paroxysmal positional vertigo; GNT-HIS, German National Telephone Health Interview Survey; HIS, International Headache Society 
Table 1 Diagnostic criteria for vestibular vertigo and benign paroxysmal positional vertigo

Vestibular vertigo (one criterion has to be fulfilled)

(1) Spontaneous rotational vertigo

(2) Positional vertigo

(3) Recurrent dizziness with nausea and either oscillopsia or imbalance

Benign paroxysmal positional vertigo (A-D have to be fulfilled)

(A) Recurrent vestibular vertigo

(B) Duration of an attack always $<1$ min

(C) Symptoms invariably provoked by the following changes of head position:

Lying down or

Turning over in the supine position

or at least 2 of the following manoeuvres:

Reclining the head

Rising up from supine position

Bending forward

(D) Not attributable to another disorder
Table 2 Population prevalence and incidence of BPPV

\begin{tabular}{lrlll}
\hline & & $\begin{array}{l}\text { Total } \\
\%(95 \% ~ C l)\end{array}$ & $\%$ Women \% Men \\
\hline $\begin{array}{l}\text { Lifetime prevalence } \\
\text { BPPV }\end{array}$ & 80 & $2.4(1.9-3.0)$ & 3.2 & 1.6 \\
$\quad$ Severe BPPV & 69 & $2.1(1.6-2.6)$ & 2.9 & 1.3 \\
$\begin{array}{l}1 \text { y prevalence } \\
\text { BPPV }\end{array}$ & 53 & $1.6(1.3-2.1)$ & 2.3 & 0.9 \\
$\quad$ 18-39y & 7 & $0.5(0.2-1.0)$ & 0.7 & 0.3 \\
$\quad 40-59$ y & 21 & $1.7(1.1-2.6)$ & 2.5 & 0.7 \\
$\quad>60$ y & 25 & $3.4(2.3-5.0)$ & 4.2 & 3.4 \\
$\begin{array}{l}4 \text { week prevalence } \\
\text { BPPV }\end{array}$ & 23 & $0.7(0.5-1.1)$ & 1.0 & 0.4 \\
Population incidence $(1$ y) & 20 & $0.6(0.4-0.9)$ & 0.8 & 0.4 \\
$\quad$ BPPV & & & & \\
\hline
\end{tabular}

BPPV, benign paroxysmal positional vertigo.

*Severe BPPV, leading to a medical consultation, interruption of daily activities or sick leave.

\section{Validation study}

For validation, telephone interviews were conducted by the neurotological survey interviewers with 61 consecutive patients from two dizziness clinics. The telephone diagnoses were compared with diagnoses of specialised neurotologists (MvB, AR, HN) after participants had presented to the clinic. The diagnosis of BPPV was made by neurotologists in 25 of 61 patients and was confirmed in 13 patients by a positive DixHallpike test, whereas in 12 patients presenting during the symptom-free interval, the diagnosis relied on a typical history and a normal neurological examination. BPPV was detected by telephone interview with a specificity of $92 \%$ and a sensitivity of $88 \%$ (positive predictive value $88 \%$, negative predictive value $92 \%)$. Telephone interviews detected migraine with a specificity of $100 \%$ and a sensitivity of $81 \%$ (positive predictive value $100 \%$, negative predictive value $84 \%$ ).

\section{Statistical analysis}

The prevalence and incidence of BPPV were calculated taking into account the two stage sampling design by multiplying the proportion of BPPV in neurotological survey participants with the proportion of dizziness/vertigo in the GNT-HIS 2003 participants. Thereby, non-responders and those lost to follow-up between the two sampling stages were assumed to have the same probability of BPPV as participants of the neurotological survey. Prevalence rates were age and sex adjusted by direct standardisation to the age and sex distribution of the German population in the year 2002. Because of the relatively small sample, the standardisation was performed using only three age groups (18-39, 40-59 and 60-79 years). The $95 \%$ confidence intervals (CI) for the prevalence and incidence estimates were calculated according to the conservative Wilson method. ${ }^{7}$ The cumulative incidence of BPPV by age was calculated for 1 year intervals by life table analysis of the GNT-HIS 2003 general population subsample which was the basis of the neurotological survey. We thereby estimated the proportion of the population that would be expected to have had BPPV by any given age. Each subject was considered to be at risk from birth until their age at interview (if unaffected) or until the age at which they developed their first episode of BPPV. Of the general population sample of 4077 GNT-HIS participants, 3819 participants were included in the life table analysis, 49 dizziness-free participants were excluded for various reasons from the final GNT-HIS dataset and were thus not available for this analysis and 209 participants with dizziness were lost to follow-up between the GNT-HIS and the neurotological survey. These 209 non-responders had an

increased probability of BPPV compared with the overall sample as we know that they had dizziness. We can therefore expect a moderate underestimation of the cumulative incidence of BPPV by this life table analysis.

The following sociodemographic factors and comorbid conditions were compared between 53 participants with BPPV in the past 12 months and a general population dizziness-free control group consisting of all participants in the GNT-HIS 2004 without vertigo/dizziness in the past 12 months $(n=6136)$ : age, sex, secondary school education (higher level, >10 years; middle level, 10 years; and lower level, <10 years), selfreported depression in the past year, smoking, body mass index, migraine according to the IHS criteria and physician diagnosed hypertension, elevated blood lipids, diabetes, coronary heart disease and stroke. The reason for choosing the GNT-HIS 2004 as a control group was that migraine according to the IHS criteria was assessed in this survey but not in the GNT-HIS 2003. The GNT-HIS 2004 belongs to the same programme of nationally representative health interview surveys as the GNTHIS 2003, has the same sampling design, a largely identical questionnaire and an almost identical response rate. Because of the uneven age and sex distribution of cases and controls, we first report odds ratios (ORs) adjusted for age and sex obtained by logistic regression models with BPPV as the outcome variable. We then performed a stepwise backward logistic regression analysis including all of the above sociodemographic variables and comorbid conditions and report the adjusted OR

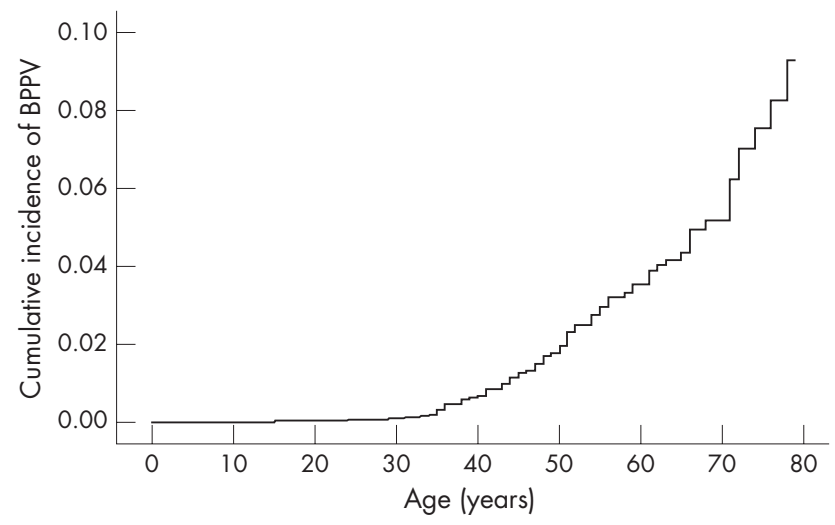

Figure 1 Cumulative incidence of benign paroxysmal positional vertigo (BPPV) by age. 
Table 3 Duration of episodes in 80 participants with benign paroxysmal positional vertigo*

\begin{tabular}{ll} 
Duration of episode & $\%$ \\
\hline$<1$ week & 45.0 \\
$1-2$ weeks & 11.2 \\
$2-4$ weeks & 12.5 \\
$4-12$ weeks & 18.8 \\
$>12$ weeks & 12.5 \\
\hline *Duration of the last episode of benign paroxysmal positional \\
vertigo.
\end{tabular}

from the final model which included sex, age, hypertension, elevated blood lipids, stroke and migraine as covariates.

\section{RESULTS}

In the neurotological survey, 243 of 1003 (24\%) participants with moderate or severe dizziness/vertigo had a history of vestibular vertigo and $80(8 \%)$ fulfilled the diagnostic criteria for BPPV ( 56 women and 24 men; aged 28-82 years). A further 23 participants $(2 \%)$ reported recurrent attacks of vestibular vertigo provoked by changes in head position with a duration of $<$ l min. These individuals were not classified as BPPV because they reported provocative head movements atypical of BPPV (ie, turning the head to the side in the upright position) $(\mathrm{n}=8)$, had less than five attacks $(n=7)$, also reported spontaneous attacks of vertigo $(n=4)$ or were considered to have a different diagnosis causing their vertiginous attacks (three participants with migrainous vertigo and one with multiple sclerosis)).

The lifetime prevalence of BPPV was $2.4 \%$, the 1 year prevalence was $1.6 \%$ and the 4 week prevalence was $0.7 \%$. The incidence of BPPV (first episode) was calculated as $0.6 \%$ per year (table 2). Prevalence and incidence rates of BPPV were consistently higher in women than in men. The 1 year prevalence of BPPV in the group of participants older than 60 years was almost seven times higher compared with that of the age group 18-39 years. Almost two thirds had experienced BPPV during the past 12 months and about $25 \%$ during the past four weeks.

Mean age of onset was 49.4 (SD 13.8) years. The age of onset was not affected by sex, secondary versus idiopathic BPPV or a history of migraine. The cumulative incidence of BPPV reached almost $10 \%$ at the age of 80 years (fig 1 ).

The median duration of the last episode of BPPV was 2 weeks (range 0.5 days to 104 weeks). One-third reported that the episode had lasted more than 1 month (table 3). The group of participants with BPPV secondary to acute unilateral vestibular loss, head trauma or intubation $(\mathrm{n}=8)$ reported a shorter duration of episodes (median 0.5 weeks) compared with participants with idiopathic BPPV (median 2 weeks). A single episode of BPPV was reported by $44 \%$ whereas $56 \%$ had recurrent episodes.

Most patients $(86 \%)$ characterised BPPV as rotational vertigo and one-third reported oscillopsia and nausea (table 4). Half of the group noticed imbalance and one-third reported fear of falling. However, actual falls during attacks of vertigo occurred in only one participant. All participants experienced BPPV in bed, most often when turning over (table 4). Restriction of head movements in order to avoid attacks of vertigo were reported by $69 \%$ of individuals. During episodes of BPPV, $24 \%$ of participants gave up driving a car and $18 \%$ avoided leaving their home. Thirty seven per cent of 54 employed BPPV patients had been on sick leave.

A medical consultation for vertigo was reported by $78 \%$ of individuals. Half of this group had consulted more than two different specialities, most often internal/general medicine $(82 \%)$, otolaryngology $(57 \%)$ and neurology $(47 \%)$. Hospital
Table 4 Clinical characteristics in 80 participants with benign paroxysmal positional vertigo

\begin{tabular}{ll}
\hline Characteristics of BPPV & $\%$ \\
Rotational vertigo & 86 \\
Oscillopsia & 31 \\
Nausea & 33 \\
Vomiting & 14 \\
Imbalance & 49 \\
Awakening due to BPPV & 49 \\
Fear of falling & 36 \\
Falls due to BPPV & 1 \\
Precipitating head movement & \\
Turning over in bed & 85 \\
Lying down & 74 \\
Rising up from supine position & 58 \\
Bending forward & 55 \\
Reclining the head & 41 \\
\hline BPPV, benign paroxysmal positional vertigo.
\end{tabular}

admission for BPPV was reported by $6 \%$ of participants. Of those participants seeking medical help, 77\% underwent laboratory testing (eg, EEG, MRI, audiogram) whereas diagnostic positioning was performed in only $27 \%$. Most individuals presenting with BPPV to a physician received no treatment $(45 \%)$ or medication for vertigo $(27 \%)$ whereas only $10 \%$ were treated with positioning manoeuvres, mostly BrandtDaroff exercises.

BPPV secondary to acute unilateral vestibular loss $(n=1)$, head trauma $(n=5)$ or intubation $(n=2)$ was identified in $10 \%$ of participants with BPPV. A history of migraine was reported by 5 men $(21 \%$ of men) and 24 women $(43 \%$ of women) (migraine without aura $\mathrm{n}=20$; migraine with aura $n=9$ ). We examined the association of BPPV with sociodemographic factors and comorbid conditions, comparing the 53 individuals with BPPV within the past 12 months with dizziness-free participants of the GNT-HIS $2004(n=6136)$. The associations tested were prespecified in the protocol: age, sex, educational level, depression, migraine, hypertension, increased blood lipids, diabetes, coronary heart disease, stroke, body mass index and smoking. After adjustment for age and sex, BPPV was associated with all of the above factors except for educational level, depression, diabetes and coronary heart disease. In a stepwise backward logistic regression model including all of these variables, only age, hypertension, increased blood lipids, stroke and migraine had an independent effect on BPPV in the past 12 months (table 5).

\section{DISCUSSION}

\section{Prevalence and incidence}

This study is the first to estimate the prevalence and incidence of BPPV in the general adult population. The lifetime prevalence was $3.2 \%$ in females, $1.6 \%$ in males and $2.4 \%$ overall. The 1 year incidence was calculated as $0.6 \%$. Based on these estimates, 1.1 million adults suffer from BPPV each year in Germany. Furthermore, BPPV is likely to be the most common vestibular disease as every third participant with vestibular vertigo had BPPV in our study.

Previous studies that aimed to assess the epidemiology of BPPV were subject to various methodological limitations and provided considerably lower incidence estimates. A study from Japan reported an incidence of BPPV of $0.01 \%$ but this can be regarded as an underestimation as only patients presenting during the acute stage to physicians affiliated with the research committee were included. ${ }^{8}$ Another study from Olmsted County, Minnesota estimated the incidence of BPPV at $0.06 \%$ on the basis of a population based medical records linkage system. However, patients not seeking medical help were not 
Table 5 Comorbidity and sociodemographic factors associated with benign paroxysmal positional vertigo*

\begin{tabular}{|c|c|c|c|c|}
\hline & \multicolumn{2}{|c|}{ Prevalence (\%) } & \multicolumn{2}{|l|}{ OR $(95 \% \mathrm{Cl})$} \\
\hline & BPPV & $\begin{array}{l}\text { Control } \\
\text { group }\end{array}$ & $\begin{array}{l}\text { Adjusted for } \\
\text { age and sex }\end{array}$ & Multivariateł \\
\hline Women & 74 & 51 & $2.4(1.3-4.5)$ & $1.8(0.9-3.4)$ \\
\hline Age (y) & & & $1.7(1.5-2.0)$ & $1.8(1.4-2.3) \top$ \\
\hline $18-39$ & 12 & 43 & & \\
\hline $40-59$ & 40 & 38 & & \\
\hline $60+$ & 48 & 19 & & \\
\hline \multicolumn{5}{|l|}{ Secondary school education } \\
\hline Higher level & 28 & 39 & 1 & \\
\hline Middle level & 29 & 35 & $1.0(0.5-2.1)$ & \\
\hline Lower level & 43 & 26 & $1.3(0.7-2.7)$ & \\
\hline Self-reported depression in the past year & 14 & 9 & $1.4(0.6-3.2)$ & \\
\hline Hypertensiont & 52 & 22 & $2.2(1.2-4.0)$ & $1.9(1.0-3.6)$ \\
\hline Elevated blood lipids $\uparrow$ & 55 & 24 & $2.5(1.4-4.4)$ & $2.0(1.1-3.7)$ \\
\hline Diabetes† & 14 & 5 & $1.6(0.7-3.8)$ & \\
\hline Coronary heart disease $\dagger$ & 18 & 5 & $2.1(0.9-4.6)$ & \\
\hline Stroke† & 10 & 1 & $6.9(1.8-19.2)$ & $4.7(2.5-13.8)$ \\
\hline \multicolumn{5}{|l|}{ Body mass index $\left(\mathrm{kg} / \mathrm{m}^{2}\right)$} \\
\hline$<25$ & 32 & 58 & 1 & \\
\hline $25-<30$ (overweight) & 52 & 33 & $2.4(1.3-4.6)$ & \\
\hline$\geqslant 30$ (obese) & 16 & 9 & $2.2(0.9-5.1)$ & \\
\hline Smoking (current daily) & 10 & 27 & $0.5(0.2-1.1)$ & \\
\hline Migraine & 34 & 10 & $7.5(3.9-14.2)$ & $8.6(4.3-17.3)$ \\
\hline \multicolumn{5}{|c|}{$\begin{array}{l}\text { BPPV, benign paroxysmal positional vertigo } \\
\text { * } 53 \text { participants with BPPV in the past } 12 \text { months compared with } 6136 \text { vertigo-free and dizziness-free participants. } \\
\text { †Self-reported physician diagnosis. } \\
\text { †Stepwise logistic regression including all variables in the table; ORs are only shown for the variables in the final model } \\
\text {-Odds ratio for increase in age per decade. }\end{array}$} \\
\hline
\end{tabular}

included and thus this study does not appear to be representative of the general population. ${ }^{9}$ Furthermore, both studies relied on a retrospective analysis of medical records which can be a source of inclusion bias. A cross-sectional study that aimed to identify unrecognised BPPV in a group of 100 unselected elderly patients with multiple chronic diseases found a strikingly high point prevalence of $9 \% .{ }^{10}$

\section{Strength and limitations of the study}

The strength of the present study was the nationwide general population setting with a large sample size and use of a validated neurotological interview with high specificity and sensitivity for the detection of BPPV. The methodological limitations of this survey are discussed. As in any interview survey, misclassification can occur as diagnostic criteria rely on a typical history of BPPV. Of note, the diagnosis of BPPV can be ultimately confirmed only when typical nystagmus is observed during positioning tests. However, this concern was partially balanced by our validation study showing that the diagnosis of BPPV can be made with high specificity and sensitivity by means of an interactive neurotological telephone interview. A limitation of the study is the self-reported nature of the comorbid factors which were included in the logistic regression model. Selection bias cannot be excluded but the nonresponder analyses which we have carried out at the different levels of non-response are reassuring. ${ }^{5}$ Willingness to participate in a further interview was not associated with the presence of dizziness or vertigo. In addition, we showed that the GNTHIS sample, which constitutes the source population for the neurotological survey, has a similar distribution of demographic characteristics and selected health indicators, as known from national statistics. ${ }^{5}$ As an exception to this, participants with a lower educational level were underrepresented in the GNT-HIS. However, we have found a lower prevalence of BPPV among those with a middle level and higher level school education and therefore our findings may even underestimate the prevalence of BPPV in the general population.
Another bias may be the fact that this study relied on recall of participants with the risk that more remote episodes tend to be more easily forgotten. This effect would be important most for the lifetime prevalence and least for the 4 week prevalence. In fact, the comparatively small difference between these rates indicates that our values for the lifetime prevalence may represent an underestimation. Furthermore, patients with BPPV may have overestimated the duration of single attacks ${ }^{11}{ }^{12}$ or may not have noticed that attacks are invariably provoked by changes in head position. Twenty-three participants with recurrent short attacks of vestibular vertigo provoked by changes in head position did not qualify for our criteria of $\mathrm{BPPV}$ for various reasons. We can speculate that a considerable proportion of this group might also have had BPPV, resulting again in an underestimation of the prevalence. Finally, a limitation of the design of this study was that we could not differentiate between BPPV of the posterior canal and variants involving other canals that can account for up to one-third of patients with BPPV. ${ }^{13}$

\section{Clinical manifestation}

The present survey has provided information on the clinical manifestations, health care use and associated comorbid factors in a cohort with BPPV, representative of the general adult population. In contrast, most previous studies on BPPV have been performed in neurotological clinics and are thus prone to selection bias. ${ }^{14-17}$

The mean age of onset of BPPV in our group was 49 years, slightly younger than in previous studies reporting a mean age of onset of 53 and 52 years. ${ }^{14}{ }^{17}$ In contrast with a previous study, ${ }^{14}$ we did not find a lower age of onset in participants with secondary compared with idiopathic BPPV but the number of participants in the former subgroup was small. Calculation of the age related cumulative incidence of BPPV shows that it starts to increase at approximately 35 years of age whereas it is very low at younger ages. 
Episodes of BPPV were mostly short with a median duration of 2 weeks. This value probably reflects the natural course of the disease as only $8 \%$ of all participants with BPPV received effective therapy. There are few studies that followed the natural course of BPPV. A prospective study has observed spontaneous remission of BPPV of the posterior canal, on average 39 days after onset, whereas in BPPV of the horizontal canal remissions occurred after 16 days. ${ }^{13}$ Previous studies may have overestimated the duration of episodes of BPPV because of selection bias as patients with rapid spontaneous remission may have been underrepresented..$^{13} 18$

Benign paroxysmal positional vertigo is a recurring disease, with a recurrence rate of approximately $15 \%$ per year. ${ }^{19}$ We found recurrence of BPPV in 56\% of individuals, which is similar to values of $50 \%{ }^{20}$ and $55 \%{ }^{21}$ reported in other studies. One limitation of our study was that the follow-up time since the first manifestation of BPPV was less than 1 year in $25 \%$ of individuals. Yet it can be concluded that BPPV has a monophasic course in less than half of patients with BPPV.

Symptoms of BPPV were characterised mostly as rotational vertigo but a small group (14\%) experienced an unspecific sensation of dizziness instead of an illusion of rotation, as previously reported in patients with BPPV confirmed by positioning testing. ${ }^{11} 12$

Furthermore, half of our group with BPPV noticed imbalance in between attacks of BPPV. Prolonged imbalance is a well known clinical feature of BPPV that can persist even after successful therapy with positioning manoeuvres ${ }^{22} 23$ and is possibly caused by utricular dysfunction. ${ }^{24}$ In the present study, only one participant with BPPV experienced falls associated with vertigo. In elderly patients with BPPV, however, falls seem to occur more often; a prevalence rate for falls of $78 \%$ has been reported in a geriatric group of patients with BPPV. ${ }^{10}$ Interestingly, all participants with BPPV described vertiginous attacks in bed, rendering this a useful screening question.

\section{Comorbid and sociodemographic factors}

We identified $10 \%$ of participants with secondary BPPV, presumably due to head trauma, acute unilateral vestibular loss or intubation. Numerous factors associated with BPPV have been reported previously, including advanced age, ${ }^{14}$ female sex,${ }^{14}$ head trauma, ${ }^{14}$ other ear disease, ${ }^{14}$ migraine, ${ }^{25}{ }^{26}$ diabetes, ${ }^{27}$ osteoporosis ${ }^{28}$ and intubation. ${ }^{14}$ Presumably, most of these conditions promote detachment of otoconia from the otolith organs whereas intubation may facilitate entry of otoconia into the posterior semicircular canal in the supine position when the head is reclined. The proportion of patients with BPPV attributed to other disorders varies between onethird and two-thirds in the literature, depending on the definition of secondary BPPV. ${ }^{14-16}$ Other inner ear diseases ipsilateral to the labyrinth affected by BPPV have been described in only $3 \%$ of patients. ${ }^{29}$ However, in these mostly uncontrolled studies, an independent or causal association of most of these factors with BPPV remains speculative.

The design of the present study allows the analysis of associations of BPPV with risk factors and comorbid conditions in an unselected group affected by BPPV. We found an association of BPPV with advanced age, with an OR of 1.8 for every decade. This is not surprising as detachment of otoconia from the otolith organs, the prerequisite for BPPV, seems to increase with age. ${ }^{30}$

We found the strongest association for BPPV with migraine. This association has been described previously. The frequency of migraine was three times higher in idiopathic BPPV than in BPPV secondary to head trauma or surgery. ${ }^{25}$ Another study found that the prevalence of migraine in patients with BPPV was twice as high as that in age and sex matched controls. ${ }^{26}$ The relationship between migraine and BPPV is poorly understood.
It has been speculated that migraine could cause vasospasm of the labyrinthine arteries, leading to detachment of otoconia from the utricular macula. ${ }^{25}$

Our study confirms previous findings of a marked female preponderance among BPPV patients. When comparing BPPV in the past 12 months with a dizziness-free control group, the age adjusted OR for female sex was 2.4 (95\% CI 1.3 to 4.5 ). When additionally adjusting for migraine, the association of female sex and BPPV was no longer statistically significant (OR $1.87 ; 95 \%$ CI 0.99 to 3.51) and remained almost unchanged after further adjustment for hypertension, blood lipids and stroke in the final model. Taking into account our sample size and the confidence interval for the adjusted OR, we conclude that the female preponderance in BPPV reflects to a considerable extent an association of migraine and BPPV but we cannot exclude an additional independent association of female sex and BPPV. The mechanism for this, however, is not known.

We found an association between BPPV and hypertension and hyperlipidaemia. To date, a link between BPPV and these vascular risk factors has not been reported or examined. Theoretically, both hypertension and hyperlipidaemia can lead to vascular damage to the inner ear and thus to BPPV. It is known that BPPV can be a sequela to labyrinthine ischaemia that probably facilitates detachment of otoconia from the otolith membrane. ${ }^{25} 31$ Furthermore, vertebrobasilar ischaemia was suggested as a predisposing factor for BPPV in several large case series. ${ }^{14-16}$ Interestingly, our data also suggest that BPPV seems to be associated with stroke. Theoretically, misclassification of participants with brainstem or cerebellar ischaemia leading to central positional vertigo could account for this finding. However, we believe that this hypothesis is rather unlikely as concomitant neurological findings were systematically requested. Of note, less than $1 \%$ of patients with ischaemia in the territory of the vertebrobasilar arteries had a single presenting symptom or sign..$^{32}$ Interestingly, age, hyperlipidaemia, hypertension and migraine ${ }^{33}$ all represent vascular risk factors and one could speculate that the common predisposing factor of BPPV might be ischaemia. However, we did not find an association between BPPV and other well established vascular risk factors such as diabetes, obesity and smoking in the multivariate analysis. A recent study showed an increased prevalence of diabetes in patients with BPPV compared with the general population..$^{27}$ This finding, however, awaits further confirmation.

\section{Psychosocial consequences}

Benign paroxysmal positional vertigo has substantial psychosocial consequences, as illustrated by our finding that BPPV led to medical consultation, interruption of daily activities or sick leave in $86 \%$ of affected individuals. Furthermore, a considerable proportion of participants avoided leaving their home or gave up driving during symptomatic episodes. The association between BPPV and depression, anxiety and diminished quality of life has been shown in several studies. ${ }^{1034-36}$ Furthermore, BPPV can lead to significant medical costs when diagnosed and treated incorrectly. In a North American study, the costs of BPPV were calculated at more than $\$ 2000$ per individual; most of these expenses were for unnecessary diagnostic measures and ineffective therapy. ${ }^{37}$ Another study from the UK calculated a mean latency between initial presentation with BPPV to the primary care physician to successful treatment of 92 weeks. ${ }^{38}$ Likewise, our study documented considerable health care utilisation in patients with BPPV. Most underwent costly laboratory tests and only a few received a correct diagnostic and therapeutic positioning. We conclude that the recommendations for diagnosis and management of this common disorder have not been effectively translated into routine clinical practice. 


\section{Authors' affiliations}

$M$ von Brevern, A Radtke, $F$ Lezius, M Feldmann, Department of Neurology, Charité, Campus Virchow-Klinkum, Berlin, Germany

T Ziese, H Neuhauser, Robert Koch Institute, Berlin, Germany

T Lempert, Department. of Neurology, Schlosspark-Klinik, Berlin, Germany

Competing interests: None.

\section{REFERENCES}

1 Brandt T. Vertigo. Its multisensory syndromes. London: Springer, 2003.

2 Neuhauser $\mathbf{H}$, Leopold $M$, von Brevern $M$, et al. The interrelations of migraine, vertigo, and migrainous vertigo. Neurology 2001;56:436-41.

3 Epley JM. The canalith repositioning procedure: for treatment of benign paroxysmal positional vertigo. Otolaryngol Head Neck Surg 1992; 107:399-404

4 Semont A, Freyss G, Vitte E. Curing the BPPV with a liberatory maneuver. Adv Otorhinolaryngol 1988;42:290-3

5 Neuhauser HK, von Brevern M, Radtke A, et al. Epidemiology of vestibular vertigo. A survey of the general population. Neurology 2005;65:898-904.

6 Headache Classification Subcommittee of the International Headache Society The international classification of headache disorders, 2 nd edn. Cephalalgia 2004;24(Suppl 1):9-160.

7 Wilson EB. Probable interference, the law of succession, and statistical interference. J Am Stat Assoc 1927;22:209-12.

8 Mizukoshi K, Watanabe $\mathrm{Y}$, Shojaku H, et al. Epidemiological studies on benign paroxysmal positional vertigo in Japan. Acta Otolaryngol 1988;447(Suppl):67-72.

9 Froehling DA, Silverstein MD, Mohr DN, et al. Benign positional vertigo: incidence and prognosis in a population-based study in Olmsted County, Minnesota. Mayo Clin Proc 1991;66:596-601

10 Oghalai JS, Manolidis S, Barth JL, et al. Unrecognized benign paroxysmal positional vertigo in elderly patients. Otolaryngol Head Neck Surg 2000;122:630-4

11 Kentala E, Pyykkö I. Vertigo in benign paroxysmal positional vertigo. Acta Otolaryngol 2000;543(Suppl):20-2

12 Yimtae K, Srirompotong S, Srirompotong S, et al. A randomized trial of the canalith repositioning procedure. Laryngoscope 2003;113:828-32.

13 Imai $T$, Ito $M$, Takeda $N$, et al. Natural course of the remission of vertigo in patients with benign paroxysmal positional vertigo. Neurology 2005;64:920-1.

14 Baloh RW, Honrubia V, Jacobson K. Benign positional vertigo: clinical and oculographic features in 240 cases. Neurology 1987; 37:371-8.

15 Katsarkas A, Kirkham TH. Paroxysmal positional vertigo-a study of 255 cases. J Otolaryngol 1978;7:320-30

16 Hughes CA, Proctor L. Benign paroxysmal positional vertigo. Laryngoscope 1997; 107:607-13.

17 Marciano E, Marcelli V. Postural restrictions in labyrintholithiasis. Eur Arch Otorhinolaryngol 2002;259:262-5.
18 von Brevern M, Lezius F, Tiel-Wilck, et al. Benign paroxysmal positional vertigo: Current status of medical management. Otolaryngol Head Neck Surg 2004;130:381-2

19 Nunez RA, Cass SP, Furman JM. Short- and long-term outcomes of canalith repositioning for benign paroxysmal positional vertigo. Otolaryngol Head Neck Surg 2000; 122:647-52.

20 Brandt T, Huppert D, Hecht J, et al. Benign paroxysmal positional vertigo: A long-term follow-up (6-17 years) of 125 patients. Acta Otolaryngol 2006; 126:160-3.

21 Korres SG, Balatsouras DG, Ferekidis E. Electronystagmographic findings in benign paroxysmal positional vertigo. Ann Otol Rhinol Laryngol $2004 ; 113: 313-18$

22 Di Girolamo S, Paludetti G, Briglia G, et al. Postural control in benign paroxysmal positional vertigo before and after recovery. Acta Otolaryngol 1998; 118:289-93.

23 Ruckenstein MJ. Therapeutic efficacy of the Epley canalith repositioning maneuver. Laryngoscope 2001;111:940-5

24 von Brevern M, Schmidt T, Schönfeld U, et al. Utricular dysfunction in patients with benign paroxysmal positional vertigo. Otol Neurotol 2006;27:92-6.

25 Ishiyama A Jacobson KM, Baloh RW. Migraine and benign positional vertigo. Ann Otol Rhinol Laryngol 2000;109:377-80.

26 Lempert $T$, Leopold $M$, von Brevern $M$, et al. Migraine and benign positional vertigo. Ann Otol Rhinol Laryngol 2000;109:1176.

27 Cohen HS, Kimball KT, Stewart MG. Benign paroxysmal positional vertigo and comorbid conditions. ORL J Otorhinolaryngol Relat Spec 2004;66:11-15.

28 Vibert D, Kompis $M$, Häusler R. Benign paroxysmal positional vertigo in older women may be related to osteoporosis and osteopenia. Ann Otol Rhinol Lanyngol 2003;112:885-9.

29 Karlberg M, Hall K, Quickert N, et al. What inner ear diseases cause beinign paroxysmal positional vertigo? Acta Otolaryngol 2000;120:380-5.

30 Igarashi M, Saito R, Mizukoshi K, et al. Otoconia in young and elderly persons: temporal bone study. Acta Otolaryngol 1993;504(Supp):26-9.

31 Lindsay JR, Hemenway WG. Postural vertigo due to unilateral sudden loss of vestibular function. Ann Otol 1956:65:692-708.

32 Savitz SI, Caplan LR. Vertebrobasilar disease. N Engl J Med 2005;352:2618-26.

33 Bousser MG, Welch KM. Relation between migraine and stroke. Lancet Neurol 2005;4:533-42.

34 Nagarkar AN, Gupta AK, Mann SBS. Psychological findings in benign paroxysmal positional vertigo and psychogenic vertigo. J Otolaryngol 2000;29:154-8.

35 Lopez-Escamez JA, Gamiz MJ, Fernandez-Perez A, et al. Long-term outcome and health-related quality of life in benign paroxysmal positional vertigo. Eur Arch Otorhinolaryngol 2005;262:507-11.

36 Magliulo G, Bertin S, Guggieri M, et al. Benign paroxysmal positional vertigo and post-treatment quality of life. Eur Arch Otorhinolaryngol 2005;262:627-30.

37 Li JC, Li CJ, Epley J, et al. Cost-effective management of benign positional vertigo using canalith repositioning. Otolaryngol Head Neck Surg 2000;122:334-339.

38 Fife D, FitzGerald JE. Do patients with benign paroxysmal positional vertigo receive prompt treatment? Analysis of waiting times and human and financial costs associated with current practice. Int J Audiol 2005:44:50-7.

\section{Keep up to date: sign up for our alerting services}

Find out automatically when an article is published on a specific topic or by a particular author. We can also alert you when an article is cited or if an eletter or correction is published. You can also choose to be alerted when a new issue is published online [and when we post articles Online First]. Check out the New Content Alerts and Citation tracker from the Online tools section on the home page. 